Journal of

Accident and

Emergency

Medicine 1994

11, 139-143

\title{
Mechanism of closed chest cardiopulmonary resuscitation investigated by transoesophageal echocardiography
}

\author{
A.C.H.PELL, ${ }^{1}$ U.M. GULY, ${ }^{2}$ G.R. SUTHERLAND, ${ }^{1}$ D.J.STEEDMAN, ${ }^{2}$ \\ P. BLOOMFIELD ${ }^{1} \&$ C. ROBERTSON ${ }^{2}$
}

Departments of ${ }^{1}$ Cardiology and ${ }^{2}$ Accident and Emergency Medicine, Royal Infirmary, Lauriston Place, Edinburgh

\section{SUMMARY}

Transoesophageal echocardiography (TOE) was performed during closed chest cardiopulmonary resuscitation (CPR) in 18 subjects in cardiac arrest. Compression of all four cardiac chambers resulted in forward flow in the pulmonary and systemic circulations, retrograde pulmonary vein flow and incomplete mitral valve closure. Antegrade pulmonary vein flow and left ventricular filling occurred exclusively during the relaxation phase. These findings support the cardiac pump theory of CPR and are incompatible with the thoracic pump mechanism. TOE merits further investigation as a device to monitor and guide resuscitation efforts during CPR.

Key words: blood flow, CPR, echocardiography mechanism

\section{INTRODUCTION}

More than $\mathbf{3 0}$ years after Kouwenhoven popularized closed chest CPR, ${ }^{1}$ the mechanisms responsible for generating forward blood flow by this technique remain uncertain. The cardiac pump theory proposes that compression of the heart between the sternum and the vertebral column results in atrioventricular valve closure and ejection of blood into the systemic and pulmonary circulations. ${ }^{1-3}$ In contrast, the thoracic pump theory suggests that the atrioventricular valves remain open during CPR, and that the left ventricle acts as a passive conduit for the passage of blood. In this model the heart is not compressed and flow is generated by pressure gradients between intrathoracic and extrathoracic vessels. $^{-4,5}$ An understanding of the mechanisms that generate blood flow during CPR has become of practical importance in light of the realization that CPR is an imperfect method of sustaining the circulation. Cardiac output may be reduced by $80 \%$ or more, myocardial perfusion may be less than $5 \%$ of normal, and successful resuscitation beyond $15 \mathrm{~min}$ of $\mathrm{CPR}$ is uncommon. ${ }^{6,7} \mathrm{~A}$ better understanding of the physiology of CPR might allow new resuscitation techniques to be developed. To elucidate these mechanisms, patients undergoing closed chest CPR were studied using TOE.

\section{METHODS}

Adults with non-traumatic, normothermic cardiac arrest, admitted to the Accident and Emergency (A\&E) Department of the Edinburgh Royal Infirmary were studied. All patients had sustained cardiorespiratory arrest in the pre-hospital setting and had received CPR and defibrillation where appropriate prior to arrival. On admission, patients were treated according to the guidelines of the UK Resuscitation Council. ${ }^{8}$ Electrical DC counter shock and antiarrhythmic agents were used as indicated. All patients were intubated and ventilated with $100 \%$ oxygen. Chest compression was administered at a rate of 80 compressions $\mathrm{min}^{-1}$, either manually or mechanically, using a Michigan Instruments Thumper Model 1004 functioning at a ratio of five chest compressions to one ventilation. ${ }^{9}$ CPR was performed with sufficient force to compress the chest $6-7 \mathrm{~cm}(36-45 \mathrm{~kg})$ and peak inspiratory pressure was set at $30 \mathrm{cms}$ of $\mathrm{H}_{2} \mathrm{O}$. The resuscitation attempt was supervised by a senior clinician who remained independent of the echocardiographic study. The study received ethical approval from the Lothian Health Board Ethics of Medical Research Committee. 
A.C.H. Pell et al.

TOE was performed using a $5 \mathrm{MHz}$ transverse plane transducer (Siemens Sonoline S11200), which was positioned in the oesophagus following tracheal intubation. Colour flow and spectral Doppler studies were performed for analysis of blood flow patterns within the heart, pulmonary veins, aorta and pulmonary artery. All studies were recorded on VHS video tape for subsequent analysis. Imaging continued until a spontaneous circulation was restored, or until the resuscitation attempt was terminated.

Echocardiographic images were studied to determine the extent of compression of the heart chambers, the timing and direction of blood flow and the patterns of valve motion. Quantitative assessment was performed using a video overlay programme (Datavue, Novamicrosonics Inc., Indianapolis, IN, USA). Frames showing the left ventricle at endrelaxation and at end-compression were selected and endocardial areas were measured with calculation of the area ejection fraction (AEF):

$$
A E F=\frac{L V A_{R}-L V A_{C}}{L V A_{R}}
$$

where $L V A_{R}$ is end-relaxation left ventricular crosssectional area and $L V A_{C}$ is end-compression left ventricular cross-sectional area.

\section{RESULTS}

\section{Study population}

Eighteen patients were studied ( 12 male, six female, age range $26-87$ years, median age 66 years). The median interval between the onset of cardiac arrest and initiation of TOE was $31 \mathrm{mins}$ (19-71 min). The initial rhythm was asystole in 10 patients, ventricular fibrillation in four patients and electromechanical dissociation in four patients. Spontaneous circulation was restored in two patients. Mechanical compression was administered to nine patients, manual compression to one patient, and both mechanical and manual compression to eight patients. Post mortem findings were available in six patients, and revealed acute coronary thrombosis $(n=5)$, and drug overdose $(n=1)$. In the remainder a clinical diagnosis of sudden cardiac death was made. The TOE probe was inserted in all patients without difficulty and did not compromise the conduct of the resuscitation attempt. There were no complications associated with TOE and none of the subjects had evidence of oesophageal trauma at

\section{General observations}

Image quality was good in all patients, and colour flow Doppler analysis of blood flow patterns within the heart and great vessels was possible in every case. Spontaneous echocardiographic contrast developed within the heart when CPR was stopped and disappeared when CPR was resumed. No differences were observed between manual and mechanical CPR.

\section{Compression phase}

The right atrial and right ventricular free walls were displaced towards the interatrial septum and interventricular septum respectively (Fig. 1), resulting in a marked reduction in cavity size (Table 1 ). This was accompanied by both tricuspid regurgitation and antegrade pulmonary artery flow. The lumen of the proximal right pulmonary artery was markedly compressed in four patients and aliasing of the colour flow Doppler signal suggested increased velocities consistent with outflow obstruction.
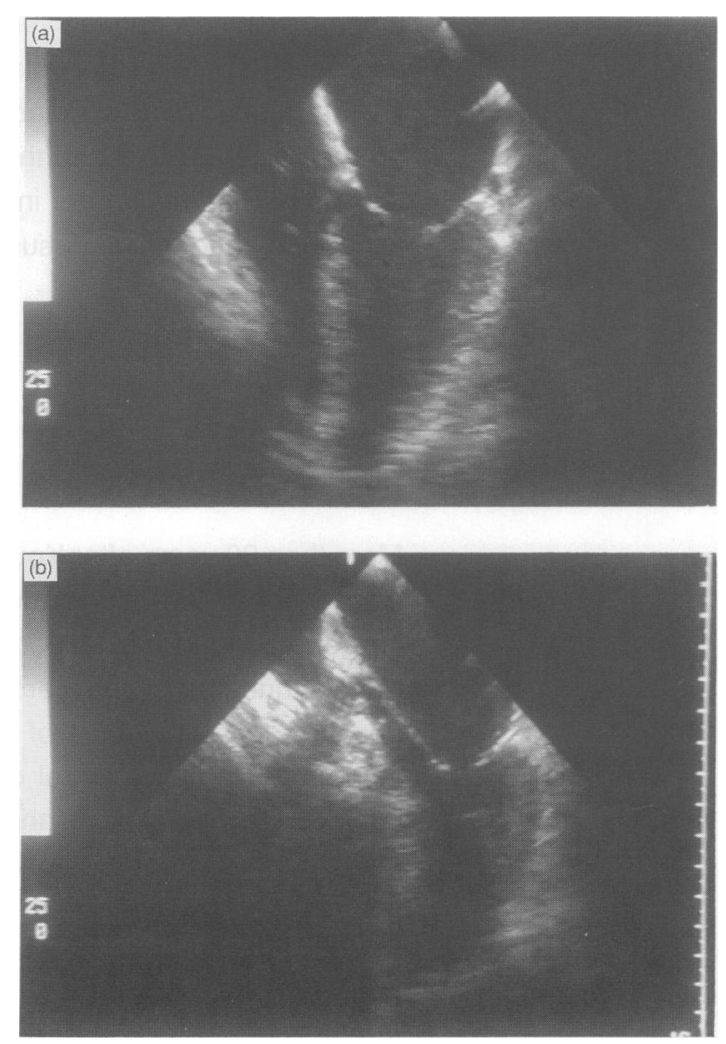

Fig. 1. Tranoesophageal echocardiographic four chamber view during relaxation (a) and compression (b) phases of CPR. There is incomplete coaptation of the mitral valve leaflets at peak compression. 
Table 1. Extent of cardiac chamber compression during CPR

\begin{tabular}{lcc}
\hline & Median area ejection fraction (\%) & Range (\%) \\
\hline RA & 62 & $49-85$ \\
RV & 74 & $39-94$ \\
LA & 23 & $10-43$ \\
LV & 33 & $19-67$ \\
\hline
\end{tabular}

Chest compression resulted in a moderate reduction in left atrial and left ventricular chamber sizes (Table 1), retrograde pulmonary vein flow (Fig. 2), antegrade flow in the left ventricular outflow tract (LVOT) and ejection of blood into the aorta. The LVOT was compressed between the interventricular septum and the anterior leaflet of the mitral valve in five patients and was associated with aliasing of the colour flow Doppler signal. Similarly, at peak compression the proximal ascending aorta and superior vena cava were also markedly compressed and distorted. Regional differences in the extent of left ventricular compression were apparent, being greater at the base of the heart than at the mid-papillary muscle level.

Mitral valve motion was biphasic. Early in the compression phase there was partial leaflet separation, but with continued compression the two leaflets approximated, although full coaptation was not usually achieved. No antegrade flow occurred across the mitral valve during chest compression, and despite incomplete valve closure, mitral regurgitation was observed in only two subjects.

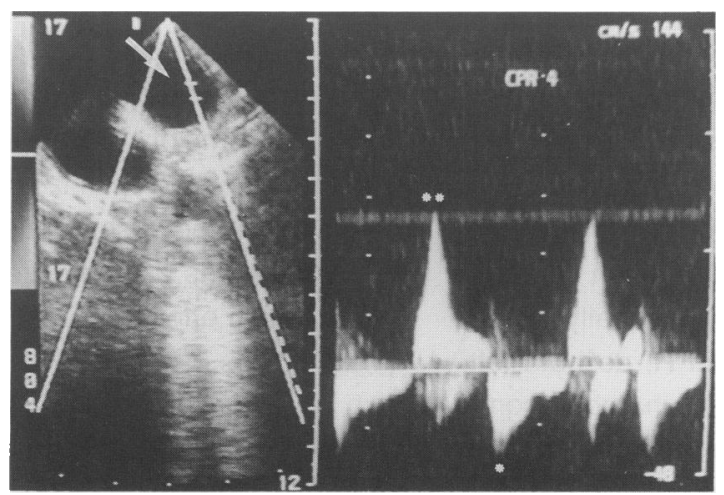

Fig. 2. Pulsed Doppler interrogation of left upper pulmonary vein (arrowed). Retrograde flow occurs during the compression phase $\left(^{*}\right)$ and antegrade flow during the relaxation phase $\left(^{\star \star}\right)$.
Relaxation phase

Immediately on release of chest compression all four heart chambers re-expanded. Flow into the right atrium from the vena cavae and coronary sinus appeared to be turbulent, and the right ventricle filled through the open tricuspid valve. There was antegrade pulmonary vein flow into the left atrium and transmitral filling of the left ventricle. Mitral valve motion was also biphasic during the relaxation phase. The valve opened initially, but partial leaflet coaptation occurred later in the phase. The aortic valve closed and there was re-expansion of the ascending aorta and superior vena cava. Mild aortic incompetence was seen in two patients. Retrograde pulmonary artery flow was apparent, but the pulmonary valve and right ventricular outflow tract were visualized poorly with TOE.

\section{Influence of underlying rhythm}

Weak contractions of both ventricles and low amplitude movements of the atrioventricular valves were observed when the underlying rhythm was electromechanical dissociation. Systolic wall thickening and change in endocardial area associated with these contractions were minimal, and colour flow Doppler revealed no effective forward flow. No spontaneous contractions occurred in ventricular fibrillation or asystole.

\section{Ineffective CPR}

In five patients the left ventricle appeared markedly hypovolaemic and chest compression caused little change in left atrial and left ventricular areas. No flow was evident with colour flow Doppler and persistence of spontaneous echocardiographic contrast suggested ineffective CPR. Post mortem results in two of these subjects revealed no underlying explanation for the hypovolaemia.

\section{DISCUSSION}

Preliminary case reports have suggested that TOE might be used to investigate the events occurring during CPR, ${ }^{10,11}$ as unlike precordial echocardiography, it can be performed continuously without compromising the resuscitation attempt. TOE is optimally positioned for visualization of both atria, the atrioventricular valves, the left ventricular outflow tract and the aortic valve, and blood flow patterns are readily demonstrated with colour flow Doppler. 
These results support the cardiac pump model as the mechanism responsible for generating forward blood flow during CPR and are consistent with a recently published echocardiographic series. ${ }^{12}$ Sternal depression caused direct compression of all four cardiac chambers and resulted in ejection of blood into the aorta and main pulmonary artery. Chamber compression was most marked on the right side of the heart, but the left atrium and ventricle were also compressed to a variable extent. In subjects with little left ventricular compression, no effective forward flow occurred.

A limitation of this study was the median delay of $30 \mathrm{~min}$ between the onset of the cardiac arrest and initiation of TOE. It has been suggested that the amplitude of mitral valve movements may diminish in prolonged resuscitation, ${ }^{13}$ and the possibility that alternative mechanisms may operate during the initial stages of CPR cannot be excluded. Nevertheless, the effectiveness of CPR was demonstrated by the restoration of sinus rhythm after prolonged resuscitation in two subjects in whom the cardiac pump mechanism was clearly responsible for the production of systemic blood flow.

Whilst the thoracic pump mechanism can operate under certain conditions, ${ }^{14}$ there was no evidence that it was responsible for the generation of forward flow in these subjects. This theory stipulates that the heart is not compressed, but that increased intrathoracic pressure is transmitted equally to all intrathoracic structures. As a result there is antegrade flow in the pulmonary veins and across the mitral valve throughout the compression phase. The evidence from TOE does not support this model. In particular, the presence of retrograde pulmonary vein flow and the absence of flow into the left ventricle during compression are incompatible with the thoracic pump mechanism.

The cardiac pump theory postulates that retrograde flow during chest compression is prevented by mitral valve closure. In this study, incomplete valve closure was observed in most subjects, yet mitral incompetence occurred in only two patients. This suggests that the pressures within the left atrium and the left ventricle may rise equally during the compression phase. During CPR, do both left heart chambers behave as a single chamber, compression of which results in ejection of blood into the aorta and retrograde pulmonary vein flow? There is limited published data on left atrial pressure changes during CPR to elucidate this issue. Similar pressures were recorded from the left atrium and femoral artery during CPR in subjects with mitral valve disease.$^{15}$ In contrast, a pressure gradient was demonstrated between the left ventricle and left atrium during CPR in dogs. ${ }^{3}$

Marked distortion of the left ventricular outflow tract, the aortic root and the right pulmonary artery commonly occurred during the compression phase and was associated with aliasing of the colour flow Doppler signal. Poor alignment from the oseophagus prevented spectral Doppler interrogation of flow at these sites, and it was not possible to ascertain whether significant outflow tract obstruction resulted. Experimentally, arterial collapse occurs when intrathoracic pressures and compressive forces are high, and this results in a reduction in carotid flow, a decreased stroke volume and the generation of a gradient between the left ventricle and the aorta. ${ }^{2,4,5}$ Whether the effectiveness of CPR in humans is limited by similar mechanisms is unclear. Indeed, compression of the ascending aorta might even contribute to forward flow if it occurred at a time when left ventricular emptying had been completed. Distortion and compression of the superior vena cava was also frequently observed during CPR. The presence of venous valves in the internal jugular veins is well recognized, ${ }^{4}$ however, compression of the superior vena cava might represent an additional mechanism by which pressure gradients are generated between the intrathoracic and extrathoracic venous systems during CPR.

TOE identified five subjects with left ventricular hypovolaemia in whom CPR was ineffective and resuscitation was unsuccessful. Left ventricular size decreases in ventricular fibrillation ${ }^{16}$ and the volume of the left ventricle may be profoundly reduced at post mortem examination. ${ }^{17}$ The pathophysiology of profound hypovolaemia is unexplained, and although it has been attributed to the position in the cardiac cycle at which the left ventricle arrests, ${ }^{17}$ extreme vasodilation is an alternative explanation. Identification of subjects in whom conventional CPR is ineffective might allow new therapeutic techniques to be developed to optimize cerebral and myocardial perfusion during resuscitation. TOE merits further investigation as a technique to monitor and guide resuscitation efforts.

\section{REFERENCES}

1. Kouwenhoven W.B., Jude J.R. \& Knickerbocker G.G. (1960) Closed-chest cardiac massage. Journal of the American Medical Association 173, 1064-1067.

2. Maier G.W., Tyson G.S., Olsen C.O. et al. (1984) The physiology of external cardiac massage: high- 
impulse cardiopulmonary resuscitation. Circulation 70, 86-101.

3. Feneley M.P., Maier G.W., Gaynor J.W. et al. (1987) Sequence of mitral valve motion and transmitral blood flow during manual cardiopulmonary resuscitation in dogs. Circulation 76, 363-375.

4. Rudikoff M.T., Maughan W.L., Effron M., Freund P. \& Weisfeldt M.L. (1980) Mechanisms of blood flow during cardiopulmonary resuscitation. Circulation 61 , 345-352.

5. Niemann J.T., Rosborough J.P., Hausknecht M., Carner D. \& Criley J.M. (1981) Pressure-synchronized cineangiography during experimental cardiopulmonary resuscitation. Circulation 64, 985-991.

6. Del Guercio L.M.R., Coomaraswany R. \& State D. Cardiac output and other hemodynamic variables during external massage in man. New England Journal of Medicine 269, 1398-1401.

7. Robertson C. \& Holmberg S. (1992) Compression techniques and blood flow during cardiopulmonary resuscitation. Resuscitation 24, 123-132.

8. UK Resuscitation Council (1984) Cardio-pulmonary Resuscitation. Laerdal Medical Limited, London.

9. Steedman D.J. \& Robertson C.E. (1990) Measurement of end-tidal carbon dioxide concentration during cardiopulmonary resuscitation. Archives of Emergency Medicine 7, 129-134.

10. Higano S.T., Oh J.K., Ewy G.A. \& Seward J.B. (1990) The mechanism of blood flow during closed chest cardiac massage in humans: transesophageal echo- cardiographic observations. Mayo Clinic Proceedings 65, 1432-1440.

11. Kuhn C., Juchems R. \& Frese W. (1991) Evidence for the 'cardiac pump theory' in cardiopulmonary resuscitation in man by transesophageal echocardiography. Resuscitation 22, 275-282.

12. Redberg R.F., Tucker K.J., Cohen T.J., Dutton J.P., Callaham M.L. \& Schiller N.B. (1993) Physiology of blood flow during cardiopulmonary resuscitation. A transesophageal echocardiographic study. Circulation 88, 534-542.

13. Deshmukh H.G., Weil M.H., Rackow E.C., Trevino R. \& Bisera J. (1985) Echocardiographic observations during cardiopulmonary resuscitation: a preliminary report. Critical Care Medicine 13, 904-906.

14. Criley I.M., Blaufuss A.H. \& Kissel G.L. (1976) Coughinduced cardiac compression. Self-administered form of cardiopulmonary resuscitation. Journal of the American Medical Association 236, 1246-1250.

15. Thomsen J.E., Stenlund R.R. \& Rowe G.G. (1968) Intracardiac pressures during closed-chest cardiac massage. Journal of the American Medical Association 205, 46-48.

16. Mashiro I., Cohn J.N., Heckel R., Nelson R.R. \& Franciosa J.A. (1978) Left and right ventricular dimensions during ventricular fibrillation in the dog. American Journal of Physiology 235, H231-H236.

17. Hutchins G.M. \& Anaya O.A. Measurements of cardiac size, chamber volumes and valve orifices at autopsy. John Hopkins Medical Journal 133, 96-106. 